\title{
Multifunctional photonic crystal barcodes from microfluidics
}

\author{
Yuanjin Zhao ${ }^{1,2,3}$, Zhuoying Xie ${ }^{1}$, Hongcheng Gu${ }^{1}$, Lu Jin ${ }^{1}$, Xiangwei Zhao ${ }^{1}$, Baoping Wang ${ }^{2}$ \\ and Zhongze $\mathrm{Gu}^{1,3}$
}

Barcode particles have a demonstrated value for multiplexed high-throughput bioassays. Attempts to develop this technology tend to focus on the generation of featured barcodes both with a large number of identifications to increase the throughput and with novel functions to improve the assays. Here, we report a new class of barcodes that are composed of multiple photonic crystal or magnetic-tagged ethoxylated trimethylolpropane triacrylate (ETPTA) cores and polyethylene glycol (PEG) hydrogel shells. These barcodes are prepared by polymerizing microfluidic multiple double emulsions. As the photonic crystal cores possess distinct reflection peaks, our barcodes allow for a substantial number of coding levels for multiplexing applications. The hydrogel shells surrounding the barcodes enable the creation of three-dimensional scaffolds for immobilizing probes. Moreover, the presence of magnetism in the barcodes confers their controllable movement under magnetic fields, which can be used to significantly increase the sensitivity of the bioassays and to simplify the processing. These features make photonic crystal barcodes ideal for biomedical applications.

NPG Asia Materials (2012) 4, e25; doi:10.1038/am.2012.46; published online 7 September 2012

Keywords: barcode; microfluidics; multiplex assay; photonic crystal

\section{INTRODUCTION}

Numerous advances in genomics and proteomics have spawned multidisciplinary efforts to develop new technologies for highthroughput multiplex assays with the ability to simultaneously detect multiple molecular targets in a single sample. Planar arrays and suspension arrays are the most significant advances with respect to multiplex assays. ${ }^{1,2}$ Planar arrays, ${ }^{3,4}$ such as nucleic acid and protein microarrays, are best suited for ultra-high-density analysis. In comparison, suspension arrays, ${ }^{5-11}$ which use barcode particles as elements, benefit from higher flexibility, fast reaction times, less reagent consumption and good repeatability in detection. Among the various suspension arrays, spectroscopic barcodes are the best established because of their simplicity in both encoding and detection. ${ }^{12-19}$ Theoretically, a large number of spectrally distinct codes can be achieved by adopting different wavelengths and intensities of the spectrum signals. ${ }^{16-19}$ However, the practicality of generated barcodes is limited because of the complicated nature of the process, which includes issues such as spectral overlapping, fluorescence quenching or bleaching and fluorescence resonance energy transfer. ${ }^{20}$ Additionally, complicated procedures are usually needed for the surface functionalization process and the probe immobilization of barcodes during the assays. Combined with the uncontrolled motion, which reduces the sensitivity of the assay and demands additional tasks for the enrichment of the decoding, these issues have limited the applications of these functions. Thus, the creation of novel barcodes with multiple, distinct advantages is anticipated.

Here, we present a new type of barcode with the desired functions based on photo-curable microfluidic multiple emulsion templates. Because of the ability to control the structures of the final emulsion, microfluidics has emerged as a promising and versatile technique for the production of monodisperse emulsions with or without multiple shell structures for different applications. ${ }^{21-27}$ However, it is still a challenge for microfluidics to generate emulsions with high-order multiple distinct cores or with the desired morphology. We overcome these limitations by employing multiple-inner phase channel capillary microfluidic devices for the generation of emulsion templates. The barcodes prepared from the templates are composed of multiple colloidal photonic crystal- or magnetic-tagged ethoxylated trimethylolpropane triacrylate (ETPTA) cores and PEG hydrogel shells. Because the photonic crystal cores possess distinct and extremely stable reflection peaks and the controversial fluorescent signal is not aroused, our barcodes allow for a remarkable spectral coding capacity in practice. Additionally, the hydrogel shells surrounding the barcodes enable the creation of functional groupenriched, three-dimensional scaffolds for immobilizing biomolecules. Moreover, from the present study of the magnetic element in the barcodes, we have determined that this approach provides excellent

\footnotetext{
${ }^{1}$ State Key Laboratory of Bioelectronics, School of Biological Science and Medical Engineering, Southeast University, Nanjing, China; ${ }^{2}$ School of Electronic Science and Engineering, Southeast University, Nanjing, China and ${ }^{3}$ Laboratory of Environment and Biosafety, Research Institute of Southeast University in Suzhou, Suzhou, China Correspondence: Professor Y Zhao or Professor Z Gu, State Key Laboratory of Bioelectronics, School of Biological Science and Medical Engineering, Southeast University, Sipailou 2, Nanjing 210096, China.

E-mail: yjzhao@seu.edu.cn or gu@seu.edu.cn

Received 21 June 2012; revised 13 July 2012; accepted 19 July 2012
} 
control over the rotation and aggregation of our barcodes under different magnetic fields, which could significantly simplify the processing of particle-based assays. These features make the photonic crystal barcodes ideal for biomedical applications.

\section{MATERIALS AND METHODS}

\section{Materials}

The materials used to prepare the outer phase were hexadecane (Sigma-Aldrich Co., Tianjin, China) with $2.5 \%$ w/w cetyl PEG/PPG-10/1 dimethicone (ABIL EM 90). The middle phase was $15 \% \mathrm{w} / \mathrm{w}$ poly(ethylene glycol) diacrylate (PEG-DA, MW 700) in water containing $0.25 \% \mathrm{w} / \mathrm{w}$ sodium dodecyl sulfate (SDS), $0.25 \% \mathrm{w} / \mathrm{w}$ ethyleneoxide propyleneoxide tri-block copolymer (Pluronic F108, BASF, Florham Park, NJ, USA), 1\% w/w methacrylic acid and 1\% w/w photoinitiator (2-hydroxy-2-methylpropiophenone, Sigma-Aldrich Co.). The inner phase was composed of a silica particle colloidal photonic crystal oil solution. To prepare the inner phase, monodisperse silica particles of various sizes were first synthesized through sol-gel chemistry by the Stober-Fink-Bohn method and seeded growth. ${ }^{28}$ Then, the prepared particles were dispersed in ethanol and mixed with ETPTA containing $0.25 \%$ v/v 2-hydroxy-2methylpropiophenone as a photoinitiator at various volume fractions. After selectively evaporating the ethanol from the mixture at $70{ }^{\circ} \mathrm{C}$ in a convection oven for approximately 1 day, the colloidal photonic crystal oil solutions were achieved; these solutions also exhibited iridescent colors. For preparing the magnetic inner phase, the synthesized silica particles $(100 \mathrm{~nm})$ and iron oxide $\left(\alpha-\mathrm{Fe}_{2} \mathrm{O}_{3}\right)$ nanoparticles (smaller than $50 \mathrm{~nm}$, Sigma-Aldrich Co.) were dispersed in ethanol and mixed with ETPTA and a photoinitiator. The amounts of silica particles and iron oxide nanoparticles were each chosen such that their weight fractions in the final, ethanol-free ETPTA were approximately $30 \%$ and $0.5 \%$, respectively. After complete mixing, the ethanol was selectively evaporated. Each prepared silica and iron oxide nanoparticle-in-ETPTA suspension was then sonicated for $30 \mathrm{~min}$ before use.

\section{Microfluidics}

The capillary microfluidic devices consist of coaxial assemblies of round and square glass capillaries on glass slides. To construct the microfluidic device for fabricating multi-core spherical double emulsions, a five-barrel capillary (5B120F-4, each barrel with an outer diameter/inner diameter ratio of 1.2 / $0.68 \mathrm{~mm}$, World Precision Instrument, Inc., Shanghai, China) was tapered to achieve an orifice diameter of approximately $50-150 \mu \mathrm{m}$ and was used for the flowing inner phases. The collection capillary tube had an outer diameter of $1 \mathrm{~mm}$ and an inner diameter of $580 \mu \mathrm{m}$. One end of the capillary was tapered to achieve a diameter of $100-300 \mu \mathrm{m}$ using a capillary puller (P-97, Sutter Instrument, Novato, CA, USA) and a microforge (MF-830, Narishige, Tokyo, Japan). The collection channel was coated with a hydrophobic reagent (trimethoxy(octadecyl)silane, Sigma-Aldrich Co.). These tapered capillaries, which were intended for the inner phase and for collecting the final emulsions, were then coaxially assembled in a square capillary with an inner diameter of $1.05 \mathrm{~mm}$ (AIT Glass, Rockaway, NJ, USA). A transparent epoxy resin was used to seal the tubes where required.

To fabricate the multi-core rod-like double emulsions, a microfluidic device comprising four inner, one middle and one outer collection glass capillary (Vitro Dynamics Inc., Rockaway, NJ, USA) was used. The inner capillaries were prepared by the heating and pulling of glass tubes (with an inner diameter of $0.3 \mathrm{~mm}$ and an outer diameter of $0.4 \mathrm{~mm}$ ), which resulted in microcapillaries with inner diameters of approximately $20 \mu \mathrm{m}$. The middle and outer capillaries possessed the same inner diameters of $100 \mu \mathrm{m}$, while the middle had a tapered tip with an inner diameter of about $70 \mu \mathrm{m}$, and the outer collection tube was treated with the hydrophobic reagent.

To generate the double emulsion templates, all fluids were pumped into the capillary microfluidic device using syringe pumps (PHD 2000 series, Harvard, Plymouth Meeting, PA, USA). During the generation of the spherical double emulsions, a typical set of flow rates for the outer, middle and inner phases was 100,1000 and $6000 \mu \mathrm{h} \mathrm{h}^{-1}$, respectively. For the rod-like double emulsions, the flow rates for the outer, middle and inner phases were 20, 100 and $100 \mu \mathrm{h} \mathrm{h}^{-1}$, respectively. The generated emulsions were polymerized in the collection channel with a UV light (S1000, OmniCure, Mississauga, Ontario, Canada) and then were collected in glass vials. The barcode particles were achieved by sequentially washing the polymerized emulsions with hexane, ethanol, water and phosphate buffer.

\section{Biomolecular assay}

For capability studies of the barcodes in biomolecule assays, oligonucleotide probes (Probe 1: $5^{\prime}-\mathrm{NH}_{2}-\left(\mathrm{CH}_{2}\right)_{6}$-TGATCGCGGTGTCAGTTCTTT-3', Probe 2 $5^{\prime}-\mathrm{NH}_{2}-\left(\mathrm{CH}_{2}\right)_{6}$-GTGGAATTGAGCAGCGTTGGT-3') and 7-amino-4-methylcoumarin (AMC)-tagged oligonucleotide targets (Target 1: 5' (AMCA)-AAAGA ACTGACACCGCGATCA-3', Target 2: 5' (AMCA)-ACCAACGCTGCTCAATTC CAC-3') were obtained from TaKaRa Biotechnology (Dalian) Co., Ltd., Dalian, China. The $10 \times$ TE buffer $(100 \mathrm{~mm}$ Tris-HCl, $10 \mathrm{~mm}$ EDTA, pH 8.0), the wash buffer $(0.2 \times$ SSC $0.2 \%$ SDS $)$ and the hybridization buffer $(750 \mathrm{~mm}$ $\mathrm{NaCl}, 150 \mathrm{~mm}$ sodium citrate, $\mathrm{pH}$ 7.4) were all prepared in-house. Oligonucleotide probes $(50 \mu \mathrm{M})$ were immobilized to the hydrogel shells of the multifunctional barcodes in the presence of coupling reagents (EDC $5 \mathrm{~mm}$, NHS $0.05 \mathrm{M}$ ) at room temperature for $3 \mathrm{~h}$ and then at $4{ }^{\circ} \mathrm{C}$ overnight. After washing the barcodes with the TE buffer and the HB buffer, they were used as the carriers for DNA hybridization in test tubes. Different concentrations of targets were used to incubate the beads $(1 \mu \mathrm{l}$ per bead). During the hybridization process, the tubes were maintained at $37^{\circ} \mathrm{C}$. The unbound oligonucleotide targets were washed away by electrophoresis $(80 \mathrm{~V}, 10 \mathrm{~min})$. The fluorescence spectra of the beads were measured after washing. For comparison, the hybridization processes were also carried out with and without rotating the magnetic field of a magnetic stirrer (IKA Laboratory Equipment, Guangzhou, China). The number of replicates at each concentration was five. The detection limit was calculated from the zero calibrator plus three times the s.d.

\section{Characterization}

The double emulsion generation processes in our capillary microfluidic device were monitored using a microscope (SMZ 745T, Nikon, Tianjin, China) Bright-field images and fluorescence images were obtained with an inverted fluorescence microscope equipped with CCD cameras (Evolution MP 5.0 Media Cybernetics, Beijing, China or DP30BW, Olympus, Nanjing, China). The photoluminescence spectra of the barcode particles were recorded by a microscope equipped with a fiber optic spectrometer (QE 65000, Ocean Optics, Hangzhou, China).

\section{RESULTS AND DISCUSSION}

In this study, multiple double emulsions were used as templates for the fabrication of the photonic crystal barcodes. These emulsions were generated with a glass capillary microfluidic device that was assembled by coaxially aligning a tapered, five-barrel capillary and a collection capillary inside a square capillary, as shown in Figure 1 and Supplementary Figure S1. The inner oil phases were pumped through the barrels of the five-barrel capillary, while the middle aqueous phase flowed through the region between the inner capillary and the outer square capillary in the same direction. The outer phase flowed through the outer capillary in the opposite direction and

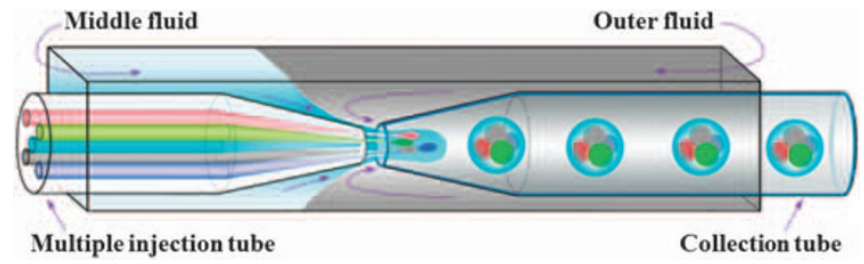

Figure $1 \mathrm{~A}$ schematic of the capillary microfluidic device used to generate the multiple core double emulsions. The injection tube has five separate internal channels, which allow four different oil phase fluids (indicated as red, green, blue and gray colors) and one aqueous phase fluid (indicated as cyan color at the center of the four oil fluids) to enter the devices separately. 
hydrodynamically focused the inner and middle phases, which broke up at the orifice of the collection tube to form monodisperse oil-inwater-in-oil $(\mathrm{O} / \mathrm{W} / \mathrm{O})$ double emulsions that contained multiple inner droplets. It is worth mentioning that although many kinds of double emulsions have been generated from microfluidics, these emulsions have only proved sufficient to encapsulate less than three kinds of different inner droplets. ${ }^{29,30}$ With our capillary microfluidic device, multiple (more than three) inner phases can be injected into the system separately; however, these inner phases tend to coalesce and form a single inner droplet during the generation of double emulsions (as shown in the Supplementary Information, Supplementary Figure S2). This behavior can be ascribed to the hydrodynamically focused inner phases by the co-flowed middle phase and flowfocused outer phase. To fabricate the double emulsions with more than three kinds of separate inner droplets, we have overcome this effect by flowing an additional aqueous phase at the center of these inner phases. Our improvement was able to prevent the coalescence of the inner droplets effectively and was able to consistently generate the microfluidic device with the desired double emulsion templates.

To impart the double emulsion templates with the potential photonic crystal characteristics, we used fluidic colloidal crystal oil solutions as the inner phases. These solutions were prepared by dispersing monodisperse silica nanoparticles in the ETPTA. ${ }^{31}$ In relatively high nanoparticle concentrations, significant interparticle repulsion occurs at the average interparticle spacing and the minimum energy configuration makes the colloidal nanoparticles self-assemble into non-close-packed face-centered cubic (FCC) or body-centered cubic colloidal crystal array structures in the medium. ${ }^{32}$ On the basis of both previous research by others ${ }^{31}$ and the following scanning electron microscopy observation results from our work, we found that the silica nanoparticles mainly formed the FCC structure in the ETPTA. Because of the periodic arrangement of the nanoparticles, the resulting hybrid ETPTA solutions possess a photonic band gap property. This property leads to the possession of certain wavelengths or frequencies located in the photonic band gap that are prohibited from propagating through the ETPTA solutions, and thus, this property imparts the solutions with vivid iridescent colors and characteristic reflection peaks (Figure 2). The main reflection peak position $\lambda$ can be estimated by Bragg's equation for a normal incident beam onto the (111) plane of the FCC structure: ${ }^{33}$

$$
\lambda=1.633 d n_{\text {average }}
$$

where $d$ is the center-to-center distance between two neighboring nanoparticles and $n_{\text {average }}$ is the average refractive index of the hybrid ETPTA solutions. Therefore, by dispersing the silica nanoparticles with different sizes or concentrations of the ETPTA solutions, the inner phases were obtained with different characteristic reflection peaks. However, a concentration of nanoparticles that is too high will hamper the ability of the inner phase droplet to form spheres, while the ETPTA with a low nanoparticle concentration exhibits poor optical quality. Thus, we prepared the hybrid ETPTA solutions with nanoparticle concentrations in the range from 20 to $30 \%$ (volume fraction). By using three kinds of silica nanoparticles with diameters of $120 \mathrm{~nm}, 160 \mathrm{~nm}$ and $200 \mathrm{~nm}$, we were also able to achieve inner phases with reflection peaks that were all in the visible light range (additional discussion in Supplementary Information).

Both the middle aqueous phase and the aqueous phase inserted at the center of the inner phases are composed of a mixture of poly(ethylene glycol) diacrylate (PEG-DA) and methacrylic acid with optimal surfactants of $0.25 \% \mathrm{w} / \mathrm{w}$ SDS and $0.25 \% \mathrm{w} / \mathrm{w}$ ethyleneoxide propyleneoxide tri-block copolymer (Pluronic F108). The outer phase
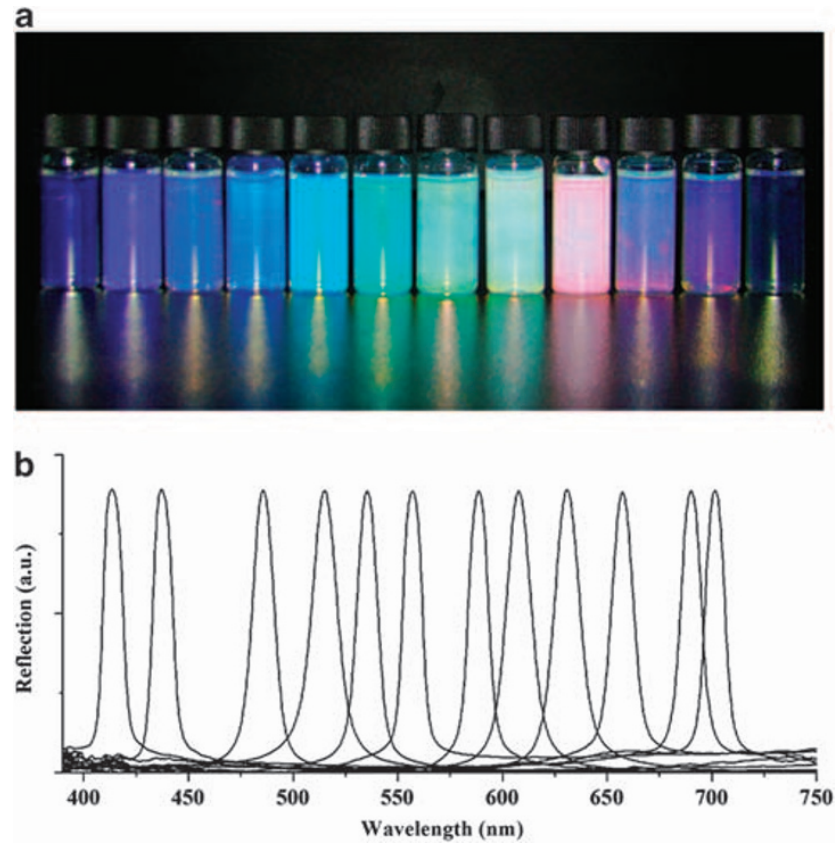

Figure 2 The silica nanoparticles-dispersed ETPTA solutions. (a) Optical images and (b) characteristic reflection peaks of the twelve kinds of photonic crystal ETPTA solutions. These solutions were prepared by using silica nanoparticles measuring 100, 120, 160 and $200 \mathrm{~nm}$ with the concentrations in the range from 20 to $30 \%$.

is hexadecane with $2.5 \mathrm{wt} \%$ of the ABIL EM 90 surfactant. Based on all the fluid phases, the double emulsions with separate inner resin droplets are fabricated. The success of the double emulsion system stems from the highly viscous nature of the hybrid ETPTA solution, which causes the crystalline photonic structure formed in the inner ETPTA droplet to be more robust to disturbances from either external flows or Brownian diffusion. By photo-polymerizing these multiple double emulsion templates with UV illumination, monodisperse photonic crystal barcodes with solid colloidal crystalline ETPTA cores (as shown by scanning electron microscopy in Figure 3) and PEG hydrogel shells are generated (Figure 4). Barcodes with different numbers and types of photonic cores could also be achieved by using different inner phases or by selectively injecting the inner phases (Figures $4 \mathrm{c}-\mathrm{f}$ ). These polymerized barcodes are highly stable and can be washed, dried and re-dispersed in water without damage.

The prepared photonic crystal barcodes have many attributes that make them an excellent choice for applications as encoded microcarriers for biomedical applications. These applications require the barcodes to have a large number of distinct identification codes for increasing throughput, a biocompatible surface for the biomolecule immobilization and reaction and a controllable motion capability for simplifying the bioassay process. Here, the photonic crystal barcodes are encoded by the multiple characteristic reflection peaks of their cores (Figure 4b), which are readily read out by a fiber optic spectrometer at a tungsten light source. Because the locations of the cores in the barcodes are random and the cores with same peak positions are undistinguishable, the number of possible photonic crystal barcodes $(N)$ can be expressed with a simple combination calculation as follows:

$$
N=\left(\begin{array}{l}
n \\
k
\end{array}\right)=\frac{n(n-1) \cdots(n-k+1)}{k(k-1) \cdots 1}
$$



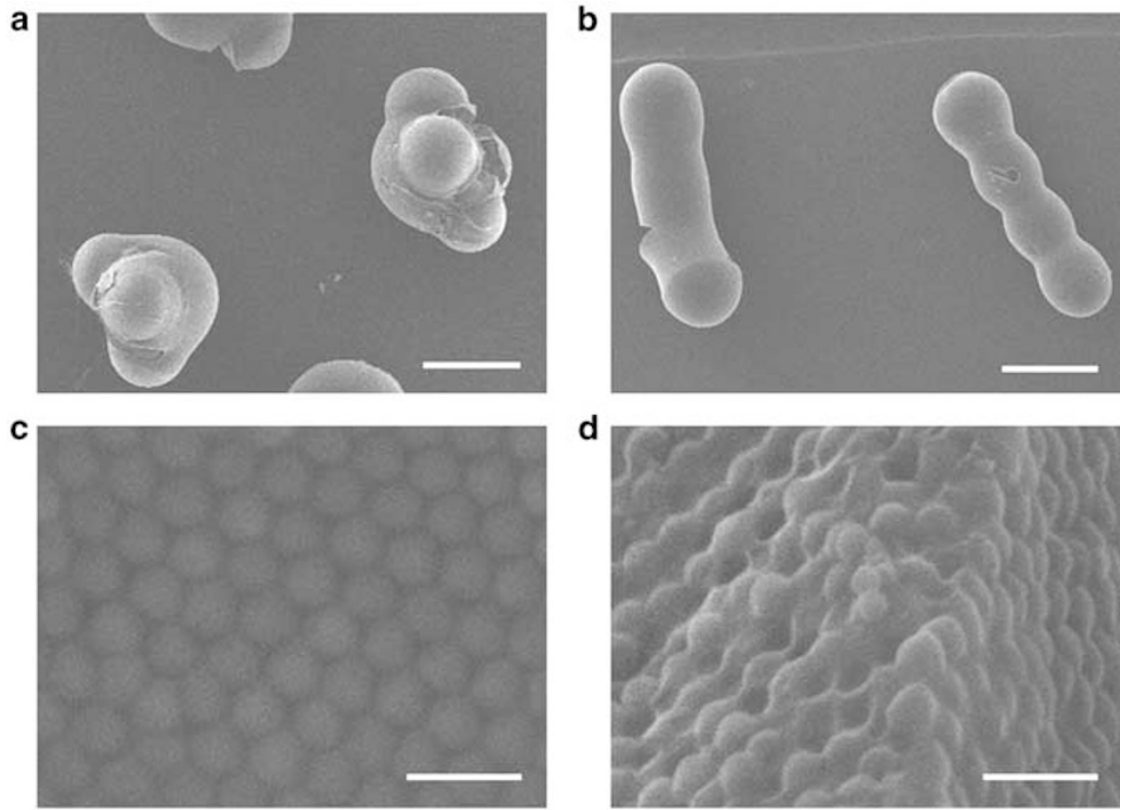

Figure 3 Scanning electron microscopy images of the barcodes. (a, b) Low-magnification images of the spherical and rod-like barcodes. (c, d) Surface and cross-sectional images of the photonic cores. Scale bars are $100 \mu \mathrm{m}$ in $(\mathbf{a}, \mathbf{b})$ and $500 \mathrm{~nm}$ in $(\mathbf{c}, \mathbf{d})$
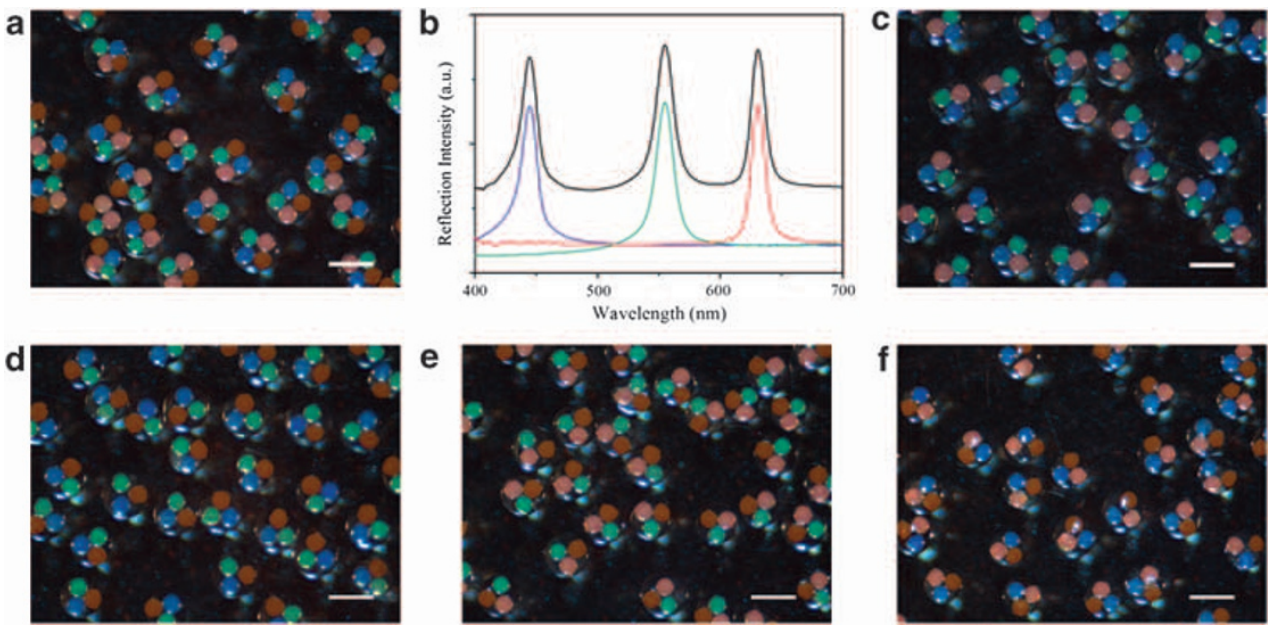

Figure 4 The barcodes with different numbers and types of photonic crystal cores (a, b). Photo and reflection spectra of four core barcodes with red, green, blue and magnetic cores (c-f). The barcodes with three different cores. Scale bars are $200 \mu \mathrm{m}$.

where $n$ is the number of all photonic crystal types with unique characteristic reflection peaks and $k$ is the number of the photonic crystal cores of the barcodes. In our research, more than 40 kinds of colloidal crystal ETPTA solutions with distinguishable reflection peaks in the visible wavelength range could be obtained. This number can be further increased by expanding the spectral range. Therefore, a total of 780 different codes can be generated by using two photonic crystal cores with 40 types of reflection peaks (that is, $n=40$ and $k=2$ ). In practice, it is realistic to use 3-4 photonic crystal cores with 60 types of reflection peaks each, yielding from 9880 to 91390 recognizable codes.

An interesting strategy to increase the identification codes can be realized by generating the barcodes with position-indexed photonic crystal cores. At this point, the rod-like double emulsions with arrayed inner droplets are ideal templates for the barcode generation. To prepare the rod-like double emulsion in a precisely controlled manner, we employed a glass capillary device composed of four inner, one middle and one outer capillary, as shown in Figure $5 \mathrm{a}$ and Supplementary Figure S3. Both the middle and outer capillaries possess the same fine-scale inner diameter. Through use of the microfluidic device, the formed inner droplets were tuned to have a diameter slightly smaller than either the middle or outer channels. The inner droplets in the middle channel and in the double emulsions of the outer channel can be well arranged in accordance with their order of formation. Because of the compression of the outer capillary, the double emulsions are able to maintain the rod-like morphology 
during flow through the outer channel. By polymerizing the double emulsion templates in the channel, we obtained the desired barcodes with position-indexed photonic crystal cores (as shown in Figures 5b-d and Supplementary Figure S4). Superior to spherical barcodes, the rod-like photonic crystal barcodes can achieve $n^{k} / 2$ recognizable codes due to the indexable and non-interfering cores, totaling to between 32000 and 1280000 identification codes of the photonic crystal barcodes by using 3-4 photonic crystal cores with 40 types of reflection peaks each. This example demonstrates the excellent capability of this encoding strategy using our photonic crystal barcodes.

To demonstrate the various capabilities of our barcodes in bioassays, we have carried out a series of proof-of-principle experiments. Typically, two kinds of barcodes with different reflection peaks were immobilized with two different oligonucleotides (probe 1 and probe 2) and were incubated in a test tube, which contained only a solution of a particular biomolecule (called target 1) for hybridization. The result indicated that the barcode reflection spectra remained constant during their application, and only the barcode with DNA probes complementary to the DNA oligomer targets showed blue fluorescence, as shown in Figure 6a. Parallel experiments were carried out by incubating the two barcodes in the test tube with target 2, both targets and no targets. In each instance, it was observed that the barcodes show high specificity to the oligomers, exhibiting fluorescence only when their corresponding target was present.

The controllable motion capability of our barcodes was integrated by inputting an additional ferric oxide-containing ETPTA solution in the inner fluidic barrel of the microfluidics during the barcode generation. Because of the anisotropic distribution of the magnetic cores in the photonic crystal barcodes, the barcode particles can be rotated under a rotating magnetic field and can be pulled to the side of a vial using a permanent magnet (as shown in Supplementray Movies S1 and S2). These features allow for the simple capturing and enrichment of the barcode particles. All encoded photonic crystal cores and magnetic cores were surrounded and stably encapsulated by the PEG hydrogel shells. This shell structure can be used to provide functional 3D scaffolds for

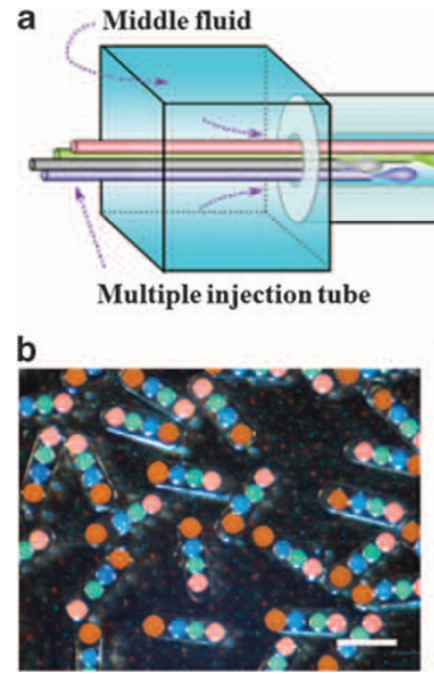

Outer fluid

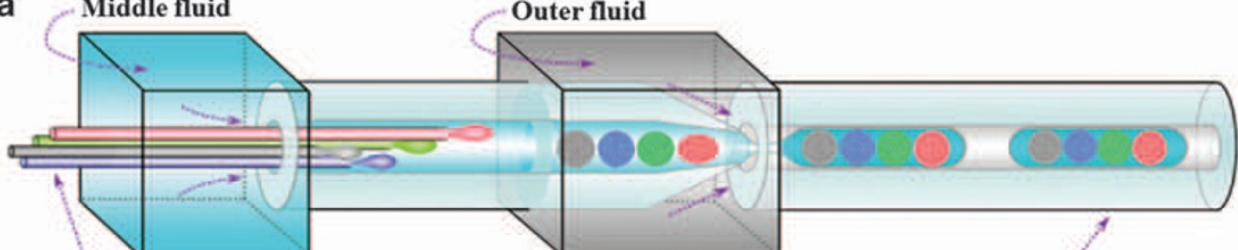

Multiple injection tube

b
C

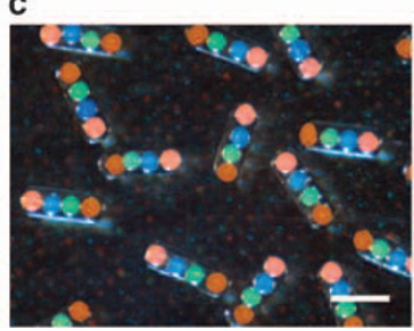

Collection tube

d

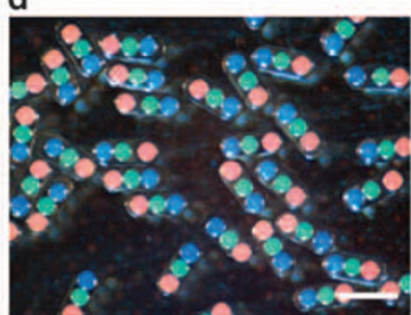

Figure 5 Rod-like photonic crystal barcodes. (a) Schematic of the capillary microfluidic device used to generate double emulsions with the rod-like barcodes. (b-d) The polymerized barcodes with the position-indexed photonic crystal cores: $(\mathbf{b}, \mathbf{c})$ the particles can be used to produce distinct codes even when the same composition of inner core is used; (d) different kinds of barcodes can also be generated by selectively injecting the oil phases of the microfluidics. Scale bars are $200 \mu \mathrm{m}$.
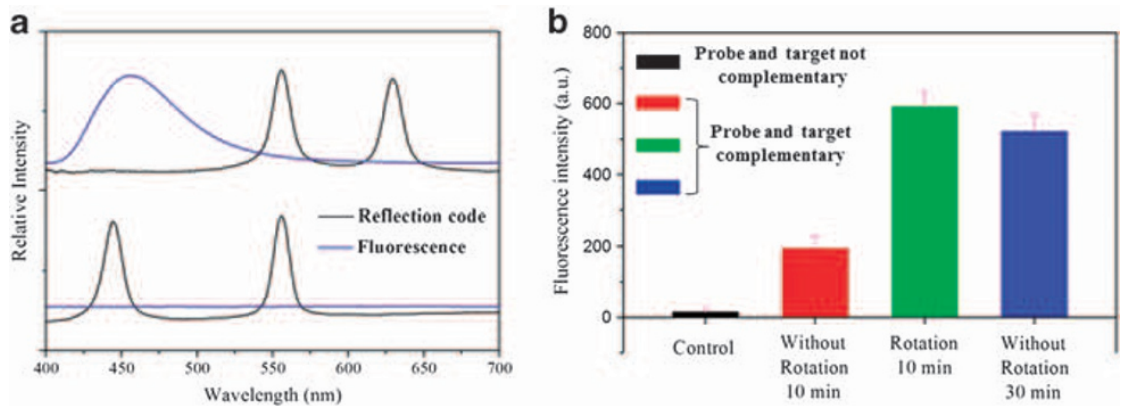

Figure 6 Barcodes for the multiplex bioassays. (a) Fluorescence (blue line) and optical reflectivity spectra (black line, background correction applied) for two kinds of barcodes in multiplex DNA assays: probe 1-loaded barcode (R555, R630) and probe 2-loaded barcode (R442, R555) were mixed together and incubated in the vessel containing target 1, thus only probe 1-loaded barcode showed a fluorescence signal after the reaction. (b) Fluorescence intensities of the barcodes after incubation with a complementary target DNA oligomer in the presence and absence of rotational motion. The error bars represent the s.e. of the measurements. 
biomolecule immobilization and for reactions with high efficiency. These scaffolds, together with the controllable motion of the barcodes, have accelerated the reaction efficiency and increased the sensitivity of our barcodes in multiplex bioassays, which have shown a threefold factor of detection signal enhancement with 10 min (Figure 6b). Therefore, our barcodes have great potential for use in high-throughput bioassays that require barcode particles with high sensitivity and controllable motion.

\section{CONCLUSIONS}

We have achieved a new class of barcode particles based on microfluidics that are composed of multiple photonic crystal or magnetic-tagged ETPTA cores and PEG hydrogel shells. The codes of our particles are determined by the characteristic reflection peaks originating in the photonic crystal cores. Because the peak position is based on their periodic structure, the code is extremely stable and the fluorescent background is not aroused. These properties render the photonic crystals an ideal encoding element for the development of barcodes. ${ }^{12,34}$ The conventional planar photonic crystal particles have achieved a large number of recognizable codes by incorporating themselves into patterned graphics. ${ }^{14}$ However, they must be properly dispersed and correctly oriented to avoid stacking or standing of the flakes during the decoding process. In comparison, photonic crystal beads, derived from the self-assembly of colloidal nanoparticles in droplets, possess spherical symmetry, and thus, they show identical photonic responses independent of their axial rotation. ${ }^{35-37}$ This behavior indicates the accurate encoding and decoding character of the photonic crystal beads. However, the possible code number of simple photonic crystal beads is practically limited by the availability of reflection peaks with distinct identification. Our newly developed barcodes possess multiple spherical photonic crystal cores; thus, their encoding element has the same high-accuracy advantage as photonic crystal beads, while their practical coding possibility is nearly exponential. Additionally, the hydrogel shells surrounding the barcodes enable the creation of functional group-enriched 3D scaffolds for immobilizing biomolecules. Moreover, the presence of a magnetic element within the photonic crystal barcodes confers excellent control over their rotation under a rotating magnetic field and aggregation under a stationary magnetic field, which could be used to significantly increase the sensitivity of particle-based assays and to simplify their processing. The unique features of our photonic crystal barcodes make them highly promising as microcarriers in biomedical applications, including high-throughput bioassays and cell culture investigations where multiplexing is needed.

\section{CONFLICT OF INTEREST}

The authors declare no conflict of interest.

\section{ACKNOWLEDGEMENTS}

We are grateful to the support of the National Science Foundation of China (Grant Nos. 50925309, 21105011, 51073034 and 51102043), the National Science Foundation of Jiangsu (Grant Nos. SBK201222247, BK2012735), the Science and Technology Development Program of Suzhou (Grant No. ZXG2012021), the Scientific Research Foundation of Southeast University (Grant Nos. Seucx201104 and 3207032104) and the 333 Talent Project Foundation. Y.J.Z. also thanks the Postdoctoral Science Foundation of China and Jiangsu province (Grant No. 1201032C).
1 Nolan, J. P. \& Sklar, L. A. Suspension array technology: evolution of the flat-array paradigm. Trends Biotechnol. 20, 9-12 (2002).

2 LaFratta, C. N. \& Walt, D. R. Very high density sensing arrays. Chem. Rev. 108, 614-637 (2008)

3 MacBeath, G. \& Schreiber, S. L. Printing proteins as microarrays for high-throughput function determination. Science 289, 1760-1763 (2000).

4 Gershon, D. Microarray technology: an array of opportunities. Nature 416, 885-891 (2002).

5 Braeckmans, K., De Smedt, S. C., Leblans, M., Pauwels, R. \& Demeester, J. Encoding microcarriers: present and future technologies. Nat. Rev. Drug Discov. 1, 447-456 (2002)

6 Wilson, R., Cossins, A. R. \& Spiller, D. G. Encoded microcarriers for high-throughput multiplexed detection. Angew. Chem. Int. Ed. 45, 6104-6117 (2006).

7 Nicewarner-Pena, S. R., Freeman, R. G., Reiss, B. D., He, L., Pena, D. J., Walton, I. D., Cromer, R., Keating, C. D. \& Natan, M. J. Submicrometer metallic barcodes. Science 294, 137-141 (2001).

8 Zhao, Y. J., Zhao, X. W. \& Gu, Z. Z. Photonic crystals in bioassays. Adv. Funct. Mater 20, 2970-2988 (2010).

9 Braeckmans, K., de Smedt, S., Roelant, C., Leblans, M., Pauwels, R. \& Demeester, J. Encoding microcarriers by spatial selective photobleaching. Nat. Mater. 2, 169-173 (2003).

10 Pregibon, D. C., Toner, M. \& Doyle, P. S. Multifunctional encoded particles for highthroughput biomolecule analysis. Science 315, 1393-1396 (2007).

11 Appleyard, D., Chapin, S. C., Srinivas, R. \& Doyle, P. S. Barcoded hydrogel microparticles for protein detection: synthesis, assay, and scanning. Nat. Protoc. 6, 1761-1774 (2011).

12 Cunin, F., Schmedake, T. A., Link, J. R., Li, Y. Y., Koh, J., Bhatia, S. N. \& Sailor, M. J. Biomolecular screening with encoded porous-silicon photonic crystals. Nat. Mater. 1, 39-41 (2002).

13 Kim, H., Ge, J. P., Kim, J., Choi, S., Lee, H., Lee, H., Park, W., Yin, Y. D. \& Kwon, S. Structural colour printing using a magnetically tunable and lithographically fixable photonic crystal. Nat. Photon 3, 534-540 (2009).

14 Lee, H., Kim, J., Kim, H., Kim, J. \& Kwon, S. Colour-barcoded magnetic microparticles for multiplexed bioassays. Nat. Mater. 9, 745-749 (2010).

15 Zhao, Y. J., Zhao, X. W., Hu, J., Li, J., Xu, W. Y. \& Gu, Z. Z. Multiplex label-free detection of biomolecules with an imprinted suspension array. Angew. Chem. Int. Ed. 48, 7350-7352 (2009).

16 Han, M. Y., Gao, X. H. Su, J. Z \& Nie, S. M. Quantum-dot-tagged microbeads for multiplexed optical coding of biomolecules. Nat. Biotechnol. 19, 631-615 (2001).

17 Wang, D. Y., Rogach, A. L. \& Caruso, F. Semiconductor quantum dot-labeled microsphere bioconjugates prepared by stepwise self-assembly. Nano. Lett. 2, 857-861 (2002)

18 Gao, X. H. \& Nie, S. M. Quantum dot-encoded mesoporous beads with high brightness and uniformity: rapid readout using flow cytometry. Anal. Chem. 76, 2406-2410 (2004)

19 Jin, Y. D., Jia, C. X., Huang, S. W., O’Donnell, M. \& Gao, X. H. Multifunctional nanoparticles as coupled contrast agents. Nat. Commun. 1, 41 (2010).

20 Wang, Z. Y., Zong, S. F., Li, W., Wang, C. L., Xu, S. H., Chen, H. \& Cui, Y. P. SERS fluorescence joint spectral encoding using organic-metal-QD hybrid nanoparticles with a huge encoding capacity for high-throughput biodetection: putting theory into practice. J. Am. Chem. Soc. 134, 2993-3000 (2012).

21 Utada, A. S., Lorenceau, E., Link, D. R., Kaplan, P. D., Stone, H. A. \& Weitz, D. A Monodisperse double emulsions generated from a microcapillary device. Science $\mathbf{3 0 8}$ 537-541 (2005)

22 Chu, L. Y., Utada, A. S., Shah, R. K., Kim, J. W. \& Weitz, D. A. Controllable monodisperse. multiple emulsions. Angew. Chem. Int. Ed. 46, 8970-8974 (2007).

23 Shah, R. K., Shum, H. C., Rowat, A. C., Lee, D., Agresti, J. J., Utada, A. S., Chu, L. Y, Kim, J. W., Fernandez-Nieves, A., Martinez, C. J. \& Weitz, D. A. Designer emulsions using microfluidics. Mater. Today 11, 18-27 (2008).

24 Shum, H. C., Zhao, Y. J., Kim, S. H. \& Weitz, D. A. Multicompartment polymersomes from double emulsions. Angew. Chem. Int. Ed. 50, 1648-1651 (2011).

25 Theberge, A. B., Courtois, F., Schaerli, Y., Fischlechner, M., Abell, C. Hollfelder, F. \& Huck, W. T. S. Microdroplets in microfluidics: an evolving platform for discoveries in chemistry and biology. Angew. Chem. Int. Ed. 49, 5846-5868 (2010)

26 Zhao, Y. J., Shum, H. C., Chen, H. S., Adams, L. A., Gu, Z. Z. \& Weitz, D. A. Microfluidic generation of multifunctional quantum dot barcode particles. J. Am. Chem. Soc. 133, 8790-8793 (2011)

27 Kim, S. H., Shim, J. W. \& Yang, S. M. Microfluidic multicolor encoding of microspheres with nanoscopic surface complexity for multiplex immunoassays. Angew. Chem. Int. Ed. 49, 3786-3790 (2010).

28 Stöber, W., Fink, A. \& Bohn, E. Growth of monodisperse silica spheres in the micron size range. J. Colloid Interface Sci. 26, 62-69 (1968).

$29 \mathrm{Kim}, \mathrm{S} . \mathrm{H}$. \& Weitz, D. A. One-step emulsification of multiple concentric shells with capillary microfluidic devices. Angew. Chem. Int. Ed. 50, 8731-8734 (2011).

30 Wang, W., Xie, R., Ju, X. J., Luo, T., Liu, L., Weitz, D. A. \& Chu, L. Y. Controllable microfluidic production of multicomponent multiple emulsions. Lab Chip 11 1587-1592 (2011)

31 Kim, S. H., Jeon, S. J., Yi, G. R., Heo, C. J., Choi, J. H. \& Yang, S. M. Optofluidic assembly of colloidal photonic crystals with controlled sizes, shapes and structures. Adv. Mater. 20, 1649-1655 (2008). 
32 Rundquist, P. A., Photinos, P., Jagannathan, S. \& Asher, S. A. Dynamical Bragg diffraction from crystalline colloidal arrays. J. Chem. Phys. 91, 4932-4941 (1989).

$33 \mathrm{Kim}$, S. H., Lee, S. Y., Yang, S. M. \& Yi, G. R. Self-assembled colloidal structures for photonics. Asia Mater 3, 25-33 (2011).

34 Zhao, Y. J., Xie, Z. Y., Gu, H. C., Zhu, C. \& Gu, Z. Z. Bio-inspired variable structural color materials. Chem. Soc. Rev. 41, 3297-3317 (2012).

35 Velev, O. D., Lenhoff, A. M. \& Kaler, E. W. A class of microstructured particles through colloidal crystallization. Science 287, 2240-2243 (2000).

36 Zhao, X. W., Cao, Y., Ito, F., Chen, H. H., Nagai, K., Zhao, Y. H. \& Gu, Z. Z. Colloidal crystal beads as supports for biomolecular screening. Angew. Chem. Int. Ed. 45, 6835-6838 (2006)
37 Zhao, Y. J., Zhao, X. W., Sun, C., Li, J., Zhu, R. \& Gu, Z. Z. Encoded silica colloidal crystal beads as supports for potential multiplex immunoassay. Anal. Chem. 80, 1598-1605 (2008).

(c) This work is licensed under the Creative Commons Attribution-NonCommercial-No Derivative Works 3.0
Unported License. To view a copy of this license, visit http:// creativecommons.org/licenses/by-nc-nd/3.0/

Supplementary Information accompanies the paper on the NPG Asia Materials website (http://www.nature.com/am) 